\title{
Non-Celiac Gluten Sensitivity in Children: Controversial Role of Gluten and Diagnostic Enigma
}

\author{
Chandra Sekhar Devulapalli *
}

Senior Medical Consultant and Pediatrician, Norwegian Labour and Welfare Administration (NAV), Medical Advisor Service, Work and Benefits Kristiania, Oslo NO-0653, Norway; E-Mail: chandev@gmail.com

* Correspondence: Chandra Sekhar Devulapalli; E-Mail: chandev@gmail.com

Academic Editor: Xia Jiang

OBM Hepatology and Gastroenterology

2021, volume 5 , issue 3

doi:10.21926/obm.hg.2103059
Received: July 07, 2021

Accepted: July 13, 2021

Published: July 22, 2021

\begin{abstract}
Non-celiac gluten sensitivity (NCGS) is a debatable condition that affects less than $6 \%$ of children. The absence of specific diagnostic markers and standardized diagnostic procedures make the diagnosis of NCGS challenging, covering patients with different and varied symptoms. Generally, the parents of small and younger children introduce a gluten-free diet (GFD) based on their personal experiences and expectations. Additionally, a "fad component" exists, contributing to the recent rise in the popularity of GFD. Thus, celiac disease (CD) and wheat allergy (WA) must be excluded as these also appear in individuals experiencing symptoms similar to those of NCGS, improving and worsening with gluten withdrawal and consumption, respectively. The role of gluten inducing gastrointestinal symptoms in individuals with self-reported NCGS has been skeptically assessed based on evidence in recent years. However, currently, it is unknown whether a strict GFD is necessary for patients with NCGS. Thus, the placebo-controlled gluten challenge remains the gold standard for a challenging diagnosis like NCGS. The present review evaluates the published studies based largely on the adult population and describes the key elements in diagnosing NCGS and differential diagnosis with CD and WA.
\end{abstract}

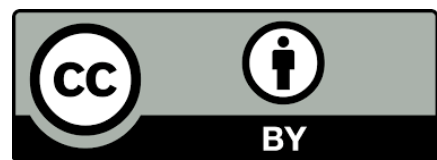

(C) 2021 by the author. This is an open access article distributed under the conditions of the Creative Commons by Attribution License, which permits unrestricted use, distribution, and reproduction in any medium or format, provided the original work is correctly cited. 


\section{Keywords}

Celiac disease; gluten; gluten-sensitive enteropathy; wheat allergy; gluten-free diet

\section{Introduction}

Non-celiac gluten sensitivity (NCGS), celiac disease (CD), and wheat allergy (WA) represent variable gluten-related disorders, although clinical manifestations observed may be similar. NCGS is defined as a clinical condition characterized by adverse gastrointestinal and extra-intestinal symptoms associated with the ingestion of gluten-or wheat-containing foods in the absence of CD or WA [1-4]. In the "classical" presentation of NCGS, gastrointestinal symptoms are similar to those of patients with irritable bowel syndrome and WA. It is suggested that NCGS can cause a wide range of extra-intestinal symptoms such as headache, migraine, "foggy mind," fatigue, fibromyalgia, irondeficiency anemia, folate deficiency, autoimmune diseases, dermatitis, atopic disorders, neurologic disorders, and psychological disorders. In children, the primary symptoms of NCGS are abdominal pain and diarrhea, while in contrast to adult patients, extra-intestinal symptoms are rare [5]. NCGS is primarily a diagnosis of exclusion in the absence of distinct diagnostic criteria $[2,6]$. In contrast, the diagnosis of $C D$ and WA is based on a combination of findings from the patient's clinical history and specific tests, including serology and duodenal biopsies for $C D$, or laboratory and functional assays for WA $[2,3,6,7]$.

The determination of the prevalence of NCGS is challenging owing to the absence of a specific diagnostic marker and standardized diagnostic procedure [2]. In a recent study, the prevalence of NCGS was 5.2\% amongst 500 patients at a pediatric clinic specializing in gluten-related disorders [8]. The authors identified $26(5.2 \%$ ) patients aged 2 - 18 years (mean $=8.42 \pm 4.1$ ) who fulfilled the clinical criteria for NCGS [8]. The patients with NCGS were predominantly boys ( $n=17,65.4 \%$ ) [8]. Although this prevalence rate is from a highly specialized population of patients with suspected gluten-related disorders [8], the true prevalence in the general population is presumed to be much lower. It varied considerably from $0.5 \%$ to $13 \%$ in a meta-analysis where 17 studies were assessed [9]; however, it included only a few children (26 of 1561 patients) who fulfilled the criteria for NCGS. Additionally, most data were from adult patients, based on patients' self-reported gluten intolerance without a medically confirmed diagnosis [9]. Therefore, it is uncertain whether these data apply to the pediatric age group. In recent years, the role of gluten causing gastrointestinal symptoms in NCGS has been the subject of evidence-based skepticism [10-12]. Considering poorly understood pathogenesis, the debatable role of gluten, and the widely popular gluten-free diet (GFD) before the diagnosis is established, the present brief review is based on emerging knowledge and attempts to clarify some of the key elements in diagnosing NCGS.

\section{Pathogenesis}

The pathogenesis of NCGS is not yet well comprehended; however, the activation of the innate immune system has been suggested $[13,14]$. There are queries regarding whether gluten or some other food components can elucidate the pathogenesis of NCGS $[13,14]$. Other proteins such as amylase trypsin inhibitors (ATIs), present in gluten-containing cereals (wheat, rye, barley, and their 
derivatives), may also demonstrate a role in the development of symptoms. Since gluten and ATIs appear simultaneously, a GFD will also be an amylase-trypsin-inhibitor-free diet [2]. In addition to gluten, fermentable oligosaccharides, disaccharides, monosaccharides, and polyols carbohydrates (FODMAPs), particularly fructans present in small quantities in gluten-containing grains, have been identified as a possible cause of some gastrointestinal symptoms in patients with NCGS [2, 14, 15]. However, whether it is gluten alone or wheat in itself (both fructans and proteins) that create a reaction is presently unknown [2]. It is also possible that other currently unknown nutrients or factors could be related to gastrointestinal symptoms of NCGS that require further elucidation. Based on the current evidence, the effect of gluten has not been proven fully in NCGS.

\subsection{Mechanisms of Gluten at the Molecular Level}

The inciting events may involve exposure of bowel epithelium to dietary gluten, further leading to immune-mediated and/or non-immune-mediated responses. Owing to the lack of evidence for $T$ cell involvement and the possible contribution from toll-like receptors (TLR-1, TLR-4), NCGS may be more of an innate rather than adaptive immune response [16]. The study by Uhde et al. [17] reported sensitivity to wheat in patients with NCGS associated with significantly increased levels of soluble CD14 and lipopolysaccharide-binding protein, as well as antibody reactivity to microbial antigens, indicating systemic immune activation. The same study demonstrated that TLR4 possibly played a role in transducing the effect of gliadin through a myeloid differentiation factor 88 adaptor protein in patients with NCGS [17]. A recent study demonstrated that the anti-gluten IgG antibody in NCGS is significantly different from CD in subclass distribution and its relationship to intestinal cell damage [18]. These findings are suggestive of a sustained primary B cell response to gluten in $C D$ and a more advanced and tolerogenic immune response to gluten in NCGS [18]. However, whether and how $B$ cell reactivity to gluten may differ in NCGS and CD, particularly regarding possible relevance to intestinal pathology, has not been investigated [18].

\section{The Controversial Role of Gluten in NCGS}

The published literature of the past ten years has demonstrated a growing tendency of evidencebased skepticism regarding the role of gluten in patients with self-reported NCGS using various adult randomized, double-blind cross-over trials. In an Australian randomized, double-blind placebocontrolled trial (DBPCT), the rate and severity of symptoms associated with placebo ingestion have been overall similar to those following gluten ingestion in those with self-reported NCGS [10]. It showed no evidence of specific or dose-dependent effects of gluten in patients with NCGS on a low FODMAP diet. A Norwegian study reported similar findings where a majority of patients with suspected NCGS using a DBPCT were unable to identify when the test involved a gluten challenge; thus, indicating that gluten was not the cause of their symptoms [11]. In contrast, fructans induced symptoms in individuals with self-reported NCGS in a randomized DBPCT conducted in Norway [12], where participants were randomly assigned to groups placed on diets containing gluten, fructans, or placebo. Following a washout period, participants crossed over into a different group until they completed all three challenges. However, the data from the aforementioned trials in adults with self-reported NCGS are not practically applicable for very young children owing to a lack of sufficient ability for both written and verbal expression [3]. Moreover, whether results from adult studies can be extrapolated to children is also uncertain, which primarily relies on unspecific symptoms. In an 
Italian study [19], only a small number of children with a positive correlation between symptoms and gluten ingestion appeared to have NCGS after a double-blind placebo-controlled gluten challenge. In this study, 1,114 children with chronic gastrointestinal symptoms (negative CD and WA); however, exhibiting a positive correlation between symptoms and gluten ingestion were eligible for a diagnostic challenge, including the following phases: run-in, open GFD, and DBPCT cross-over gluten challenge. Of these, $96.7 \%$ did not exhibit any correlation with gluten ingestion. Thirty-six children were eligible; after the run-in and open GFD, 28 patients entered the gluten challenge. There were 11 children (39.2\%) who tested positive. Children in this study were randomized to gluten ( $10 \mathrm{~g} /$ daily) and placebo (rice starch) each for two weeks, segregated by a washout week. The gluten challenge was considered positive when there was a minimum $30 \%$ decrease of the global visual analog scale between gluten and placebo. The results of the mentioned study [19] reinforced the requirement of a DBPCT for the diagnosis. Thus, the aforementioned clinical trials reflect a possibility that gastrointestinal symptoms in patients with NCGS are often wrongly attributed to dietary gluten. However, it is uncertain whether extra-intestinal symptoms that are observed in patients with NCGS are related to the ingestion of gluten-containing food that needs further investigation.

\section{Diagnostic Challenges}

The diagnosis of NCGS should be considered in patients with persistent intestinal and/or extraintestinal complaints showing a normal result of the CD and WA serological markers on a glutencontaining diet, generally reporting worsening of symptoms after eating gluten-containing food [1]. The confirmation of the diagnosis of NCGS should aim to: (1) assess the clinical response to the GFD; (2) measure the effect of reintroducing gluten after GFD treatment. However, many of these patients are already following the GFD at the time of reporting. In contrast to CD and WA, the diagnosis of NCGS is more challenging as there are no specific clinical biomarkers and owing to relatively poorly comprehended pathogenesis. A large proportion of patients are self-diagnosed and initiate a GFD without a sufficient clinical basis, further posing a challenge. The European Society for Paediatric Gastroenterology Hepatology and Nutrition guidelines recommend a complete clinical and laboratory evaluation to exclude CD and WA while still on a gluten-containing diet [7]; however, a biopsy to exclude the diagnosis of CD is not required [7]. In contrast, the European Society for the Study of Celiac Disease guidelines for adults recommends a duodenal biopsy when highly suspicious of CD [16]. However, probably many adults and children are advised unnecessary elimination diets without adequate clinical assessment [2,3]. NCGS can only be diagnosed using a double-blind, placebo-controlled, dietary cross-over challenge with gluten [1-4]. It provides the most accurate results when the parents or patients strongly expect a reaction and for subjective symptoms [20]. It is generally performed in research studies as it is considered expensive and complicated for routine clinical use [4]. However, it is a useful tool to exclude a placebo effect and the best available alternative for a challenging and controversial diagnosis like NCGS [19, 20]. Although only a minority of patients with self-reported gluten sensitivity demonstrate reliable and reproducible symptoms $[4,21]$, these should be identified as there are financial consequences for the patients. The cost of this test is outweighed by the benefits to patients' wellbeing and the unnecessary financial burden of a GFD [20]. 
It is plausible that the current criteria used for diagnosing NCGS may also mistakenly include some with latent or subclinical CD. The onset of NCGS symptoms may be delayed from few hours to a few days after gluten ingestion, whereas in $C D$, it can take days to weeks [22, 23]. As per the definition of NCGS, serological markers of CD (IgA tissue transglutaminase, IgA endomysial antibody, and IgG deaminated gliadin peptide antibodies) are always negative in patients with NCGS. However, some patients with NCGS may have CD [9]. A systematic review in 2015 demonstrated that $20 \%$ of people with NCGS presenting with human leukocyte antigen (HLA)-DQ2 and/or HLA-DQ8 haplotypes, negative serology, and normal histology or duodenal lymphocytosis had CD [9]. However, the incidence of $20 \%$ of individuals with CD-associated HLA is similar or less than expected since this HLA-type is present in approximately $30 \%$ of the general population. In those with CD before diagnosis who are already on a gluten-containing diet, serological markers for $C D$ are not consistently detected [22]. Moreover, duodenal biopsies in individuals with NCGS are consistently almost normal [15, 24-26], which is an essential parameter for NCGS diagnosis. However, a subgroup of people with NCGS may have an increased number of duodenal intraepithelial lymphocytes ( $\geq$ 25/100 enterocytes), which represent Marsh I lesions [9].

In contrast to NCGS, WA has a fast onset (from minutes to hours) after the consumption of food containing wheat and possibly leading to anaphylaxis $[22,27]$. In most cases, the clinical presentation may be sufficient to distinguish a WA from other entities [26]. WA is excluded when there are normal levels of serum IgE antibodies to gluten proteins and wheat fractions and no skin reaction to prick tests for WA [23]. If an allergic reaction cannot be identified, the diagnosis should be confirmed using double-blinded and placebo-controlled food provocation tests [20]. Delayed allergic reactions may occur with these types of tests; however, currently, there is no international consensus statement on diagnosing delayed wheat-related symptoms. When symptoms are limited to gastrointestinal effects, there may be an overlap with WA and irritable bowel syndrome [28]. However, the presence of related extra-intestinal manifestations has been suggested to be a feature of NCGS [28].

\subsection{Management of NCGS}

After establishing an NCGS diagnosis, a GFD is initiated to assess if symptoms improve or are resolved completely. This may occur within days to weeks of starting a GFD; however, improvement may also be due to a non-specific, placebo response. Although $C D$ requires adherence to a strict lifelong GFD, whether NCGS is a permanent or a transient condition is unknown [23, 27]. A trial of gluten reintroduction to observe any reaction after 1-2 years of a strict GFD might be performed [23]. There is an increased consumption of gluten-free foods in the United States over the last three decades, disproportionate to the prevalence of gluten-related disorders [29]. Similarly, in recent years, additional food expenses due to gluten-related disorders have primarily granted basic benefits in Norway [30]. Gluten-free eating patterns are frequently perceived to be healthier than gluten-containing ones, and GFD has emerged as a lifestyle statement in recent years. However, subjective feelings of improvement after GFD does not indicate it to be healthier than glutencontaining food items. A recent study demonstrated that gluten-free eating patterns do not have healthier macronutrient or micronutrient profiles, apart from lower sodium levels [31]. On the contrary, gluten-free foods are less optimal for dietary fiber, folate, total protein, vitamin E, magnesium, and potassium [31]. Moreover, in the absence of medical indications to restrict gluten 
consumption, the potential for lower fiber and several limiting micronutrients highlights the potential for negative effects of gluten-free dietary patterns on long-term health [31]. It appears that often self-diagnosed patients initiate and follow a GFD without thorough assessment by a specialist [3]. The primary challenge for the clinician is an inability to exclude CD in this setting. Placebo-controlled gluten challenge is required in patients responding to treatment using the GFD and in those who are already on a GFD before testing and are willing to establish the diagnosis [1, $16,20]$.

\section{Conclusions}

The published literature suggests that we are at a nascent stage of understanding NCGS. Whether innate or adaptive immune responses are involved in NCGS or the condition relates specifically to gluten or some other components of grains has not been established. Thus, future clinical research should address and determine more precisely the role of gluten and other nutrients. Furthermore, the diagnostic techniques for detecting NCGS must be improved, and clear biomarkers should be developed. Until we find distinguishing biomarkers (clinical, biochemical, and histopathological findings) that specifically support the condition of NCGS, the diagnosis would probably be enhanced by including routine use of gluten challenge in clinical practice [20]. Currently, the role of gluten is controversial in patients with self-reported NCGS, and gastrointestinal symptoms are often wrongly attributed to dietary gluten. However, gluten avoidance in the general population appears to be more like a possibility than a necessity and reflects a certain "healthy" lifestyle without necessarily indicating a medical need.

\section{Author Contributions}

The author did all the research work of this study.

\section{Competing Interests}

The authors have declared that no competing interests exist.

\section{References}

1. Catassi C, Elli L, Bonaz B, Bouma G, Carroccio A, Castillejo G, et al. Diagnosis of non-celiac gluten sensitivity (NCGS): The Salerno experts' criteria. Nutrients. 2015; 7: 4966-4977.

2. Devulapalli CS. Non-coeliac gluten sensitivity in children. Tidsskr Nor Laegeforen. 2020; 140. doi: 10.4045/tidsskr.19.0777.

3. Devulapalli CS. Gluten-free diet in children: A fad or necessity? Arch Dis Child. 2021; 106: 628629.

4. Potter M, Walker MM, Talley NJ. Non-coeliac gluten or wheat sensitivity: Emerging disease or misdiagnosis? Med J Aust. 2017; 207: 211-215.

5. Ruemmele FM. Non-celiac gluten sensitivity: A challenging diagnosis in children with abdominal pain. Ann Nutr Metab. 2018; 73: 39-46.

6. Elli L, Branchi F, Tomba C, Villalta D, Norsa L, Ferretti F, et al. Diagnosis of gluten related disorders: Celiac disease, wheat allergy and non-celiac gluten sensitivity. World J Gastroenterol. 2015; 21: 7110-7119. 
7. Husby S, Koletzko S, Korponay-Szabó I, Kurppa K, Mearin ML, Ribes-Koninckx C, et al. European society paediatric gastroenterology, hepatology and nutrition guidelines for diagnosing coeliac disease 2020. J Pediatr Gastroenterol Nutr. 2020; 70: 141-156.

8. Camhi SS, Sangal K, Kenyon V, Lima R, Fasano A, Leonard M. Pediatric nonceliac gluten sensitivity: A gluten-related disorder treatment center experience. J Pediatr Gastroenterol Nutr. 2019; 69: 200-205.

9. Molina-Infante J, Santolaria S, Sanders DS, Fernández-Bañares F. Systematic review: Noncoeliac gluten sensitivity. Aliment Pharmacol Ther. 2015; 41: 807-820.

10. Biesiekierski JR, Peters SL, Newnham ED, Rosella O, Muir JG, Gibson PR. No effects of gluten in patients with self-reported non-celiac gluten sensitivity after dietary reduction of fermentable, poorly absorbed, short-chain carbohydrates. Gastroenterology. 2013; 145: 320-328.e3.

11. Dale HF, Hatlebakk JG, Hovdenak N, Ystad SO, Lied GA. The effect of a controlled gluten challenge in a group of patients with suspected non-coeliac gluten sensitivity: A randomized, double-blind placebo-controlled challenge. Neurogastroenterol Motil. 2018; 30: e13332.

12. Skodje GI, Sarna VK, Minelle IH, Rolfsen KL, Muir JG, Gibson PR, et al. Fructan, rather than gluten, induces symptoms in patients with self-reported non-celiac gluten sensitivity. Gastroenterology. 2018; 154: 529-539.e2.

13. Fasano A, Sapone A, Zevallos V, Schuppan D. Nonceliac gluten sensitivity. Gastroenterology. 2015; 148: 1195-1204.

14. Leonard MM, Sapone A, Catassi C, Fasano A. Celiac disease and nonceliac gluten sensitivity: A review. JAMA. 2017; 318: 647-656.

15. Ontiveros N, Hardy MY, Cabrera-Chavez F. Assessing of celiac disease and nonceliac gluten sensitivity. Gastroenterol Res Pract. 2015; 2015: 1-13.

16. Al-Toma A, Volta U, Auricchio R, Castillejo G, Sanders DS, Cellier C, et al. European Society for the Study of Coeliac Disease (ESSCD) guideline for coeliac disease and other gluten-related disorders. United European Gastroenterol J. 2019; 7: 583-613.

17. Uhde M, Ajamian M, Caio G, De Giorgio R, Indart A, Green PH, et al. Intestinal cell damage and systemic immune activation in individuals reporting sensitivity to wheat in the absence of coeliac disease. Gut. 2016; 65: 1930-1937.

18. Uhde M, Caio G, De Giorgio R, Green PH, Volta U, Alaedini A. Subclass profile of IgG antibody response to gluten differentiates nonceliac gluten sensitivity from celiac disease. Gastroenterology. 2020; 159: 1965-1967.

19. Francavilla R, Cristofori F, Verzillo L, Gentile A, Castellaneta S, Polloni C, et al. Randomized double-blind placebo-controlled crossover trial for the diagnosis of non-celiac gluten sensitivity in children. Am J Gastroenterol. 2018; 113: 421-430.

20. Devulapalli CS. The gluten challenge is still the best option for children with a difficult diagnosis like non-coeliac gluten sensitivity. Acta Paediatr. 2021;110: 1104-1106.

21. Potter MD, Walker MM, Keely S, Talley NJ. What's in a name? 'Non-coeliac gluten or wheat sensitivity': Controversies and mechanisms related to wheat and gluten causing gastrointestinal symptoms or disease. Gut. 2018; 67: 2073-2077.

22. Fasano A, Catassi C. Clinical practice. Celiac disease. N Engl J Med. 2012; 367: 2419-2426.

23. Volta U, Caio G, De Giorgio R, Henriksen C, Skodje G, Lundin KE. Non-celiac gluten sensitivity: A work-in-progress entity in the spectrum of wheat-related disorders. Best Pract Res Clin Gastroenterol. 2015; 29: 477-491. 
24. Aziz I, Hadjivassiliou M, Sanders DS. The spectrum of noncoeliac gluten sensitivity. Nat Rev Gastroenterol Hepatol. 2015; 12: 516-526.

25. Green PH, Lebwohl B, Greywoode R. Celiac disease. J Allergy Clin Immunol. 2015; 135: 10991106

26. Tonutti E, Bizzaro N. Diagnosis and classification of celiac disease and gluten sensitivity. Autoimmun Rev. 2014; 13: 472-476.

27. Vriezinga SL, Schweizer JJ, Koning F, Mearin ML. Coeliac disease and gluten-related disorders in childhood. Nat Rev Gastroenterol Hepatol. 2015; 12: 527-536.

28. Schuppan D, Pickert G, Ashfaq-Khan M, Zevallos V. Non-celiac wheat sensitivity: Differential diagnosis, triggers and implications. Best Pract Res Clin Gastroenterol. 2015; 29: 469-476.

29. Kim HS, Patel KG, Orosz E, Kothari N, Demyen MF, Pyrsopoulos N, et al. Time trends in the prevalence of celiac disease and gluten-free diet in the US population: Results from the National health and nutrition examination surveys 2009-2014. JAMA Intern Med. 2016; 176: 1716-1717.

30. Devulapalli CS. Basic benefit in the case of non-coeliac gluten sensitivity. Tidsskr Nor Laegeforen. 2020; 140. doi: 10.4045/tidsskr.20.0581.

31. Taetzsch A, Das SK, Brown C, Krauss A, Silver RE, Roberts SB. Are gluten-free diets more nutritious? An evaluation of self-selected and recommended gluten-free and gluten-containing dietary patterns. Nutrients. 2018; 10: 1881.

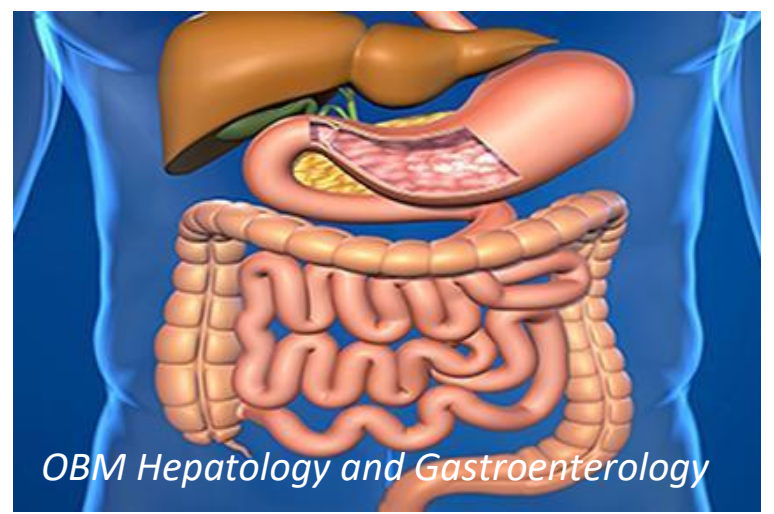

Enjoy OBM Hepatology and Gastroenterology by:

1. Submitting a manuscript

2. Joining in volunteer reviewer bank

3. Joining Editorial Board

4. Guest editing a special issue

For more details, please visit: http://www.lidsen.com/journals/hg 